\title{
Der Lipödemschmerz und seine Folgen auf die Lebensqualität betroffener Patientinnen - Ergebnisse einer Patientenbefragung mittels Schmerzfragebogen
}

\section{Pain in lipoedema, fat in lipoedema and its consequences: results of a patient survey based on a pain questionnaire}

Autoren

Matthias H.L. Gensior, Manuel Cornely

Institut

CG-Lympha, Fachklinik für operative Lymphologie, Köln

Schlüsselwörter

schmerzhaftes Fettgewebe, komplexes regionales

Schmerzsyndrom, Lebensqualität

Key words

local pain syndrome, quality of life in lipoedema,

pain in adipose tissue

eingereicht 18.10.2018

akzeptiert 13.05.2019

online publiziert 26.02 .2020

\section{Bibliografie}

Phlebologie 2020; 49: 330-335

DOI 10.1055/a-1109-5960

ISSN 0939-978X

(c) 2020. Thieme. All rights reserved.

Georg Thieme Verlag KG, Rüdigerstraße 14,

70469 Stuttgart, Germany

Zitierweise für diesen Artikel Handchir Mikrochir Plast

Chir 2019; 51(04): 249-254

Korrespondenzadresse

Dr. Matthias H.L. Gensior

CG Lympha GmbH, Fachklinik für operative Lymphologie,

Gereonstraße 18-32, 50670 Köln

m.b.gensior@online.de

\section{ZUSAMMENFASSUNG}

Bei 640 Patientinnen einer Fachklinik für operative Lymphologie erfolgte mittels Fragebogen der Deutschen Schmerzgesellschaft e. V. eine Befragung. Neben Fragen zum Schmerz und zur Schmerzcharakteristik wurden gleichzeitig noch demografische Daten miterhoben. Es ergab sich, dass nur bei etwas über $50 \%$ eine echte Adipositas nachgewiesen werden konnte. Lipödem und Adipositas müssen als unabhängige

Krankheitsbilder gewertet werden. Der Schmerz wurde überwiegend als drückend und ziehend empfunden. Attribute wie klopfend oder pochend, passend zu einer akuten Entzündung, erfuhren die Wertung „nicht zutreffend“. Die Beschwerdesymptomatik war unabhängig vom BMI, der bei der Lipohyperplasie dolorosa nur bedingt verwertbar ist. Insgesamt ist das Leitsymptom „Schmerz" sehr facettenreich, das angeborene, nicht erworbene Lipödemfett der Extremitäten führt zu einer deutlichen Beeinträchtigung der Aktivitäten sowohl allgemein als auch im Freizeitbereich. Die durch den G-BA initiierte Studie muss daher kritisch gesehen werden. Da bislang keine objektivierbaren Befunde beim Lipödem erhoben werden können, ist eine subtile Befragung betroffener Patientinnen zur Diagnosestellung notwendig.

\section{ABSTRACT}

640 patients from a specialist clinic for operative lymphology were surveyed with the help by a questionnaire issued by the German Society of Pain Therapy (Deutsche Schmerzgesellschaft e. V.). This survey collected responses to questions about pain and pain characteristics as well as demographic data. It revealed that only a little more than $50 \%$ of respondents were genuine cases of obesity. Lipoedema and obesity must therefore be regarded as clinical pictures unrelated to one another. The pain was mostly described as pressing and tearing in nature. Attributes such as throbbing or pulsing, consistent with acute inflammation, were rated as "not applicable". Symptoms were independent of the BMI, which is only useable to a limited extent in lipohyperplasia dolorosa. On the whole, the main symptom "pain" is multi-faceted. The study initiated by the German Federal Joint Committee (G-BA) must therefore be viewed critically. Congenital (as opposed to acquired) lipoedema fat on the extremities significantly impairs a person's ability to undertake activities in general as well as leisure activities. Since no objectively verifiable findings in lipoedema can be ascertained thus far, the diagnosis should be based on a careful patient survey. 


\section{Einleitung}

Das Lipödem ist eine chronische und progrediente Erkrankung, die fast ausschließlich bei Frauen auftritt und durch eine Fettverteilungsstörung mit deutlicher Disproportion zwischen Körperstamm und den Extremitäten gekennzeichnet ist [1].

Die erstmalige Beschreibung dieses Krankheitsbildes erfolgte durch Allen und Hines im Jahr 1940 [2]. Dieses Krankheitsbild wurde zunächst wenig beachtet. Erst im Verlauf der 1990er-Jahre rückte dieses Krankheitsbild in den Fokus von Ärzten und auch Patienten [3]. Das klinische Erscheinungsbild ist sehr facettenreich, wird aber durch folgende Kriterien charakterisiert ( $\triangleright$ Tab. 1):

Trotz zunehmender Forschungstätigkeit liegen bislang nur wenig gesicherte Erkenntnisse bzgl. Ursache und objektivierbaren Befunden vor [4]. Auch in der neuen Literatur finden sich nur wenige gesicherte Erkenntnisse sowohl zur Pathophysiologie als auch Untersuchung [5-7]. Speziell in den Stadien I und II sind Untersuchungen wie Ultraschalluntersuchungen, Darstellung der Lymphgefäße mittels Farbstoffinjektion und Infrarotlicht unspezifisch. Auch ein reguläres Funktionslymphszintigramm zeigt in der Regel nur ein gut funktionierendes orthologes Lymphgefäßsystem an. Quantitative Messungen des Lymphflusses konnten einen Hochvolumentransport nachweisen [8]. Hieraus wurde eine Hochvolumen-Transportinsuffizienz abgeleitet, die zu dem von Marsch und Cornely beschriebenen pathophysiologischen Grundprozess der Lipohyperplasia dolorosa [9], also des Lipödems, führt [10-12]. Cornely prägte später den Begriff der „Marsch'en These“. Die Diagnosestellung dieses Krankheitsbildes erfolgt jedoch nach wie vor in erster Linie abhängig vom klinischen Erscheinungsbild. Als eines der Leitsymptome dieser Erkrankung gilt jedoch der Schmerz. Eine Untersuchung zur Charakterisierung des Schmerzbildes wurde 2008 von Schmeller und Meier-Vollrath [13] durch einen selbst erstellten Fragebogen vorgenommen. Im Rahmen dieser Untersuchung wurden 50 Patientinnen ausschließlich mit einem Lipödem im Stadium II untersucht. Dabei wurde der Schmerz in erster Linie als dumpf und drückend angegeben. In einer Vielzahl von Veröffentlichungen wurde versucht, zum einen das Schmerzbild zu charakterisieren, zum anderen eine Ursache für die Schmerzhaftigkeit bei diesem Krankheitsbild zu finden. Brenner hat 2017 hierzu eine Übersichtsarbeit veröffentlicht [14]. Fazit seiner Recherche und Publikation ist, dass bislang weder eine Beschreibung noch eine Erklärung für die Schmerzsymptomatik möglich ist. Auch eine Charakterisierung des Schmerzbildes bei Lipödem-Patientinnen konnte nicht vorgenommen werden.

Trotz dieser bestehenden Unsicherheit bzgl. des Schmerzbildes beim Lipödem hat der gemeinsame Bundesausschuss der gesetzlichen Krankenkassen (G-BA) in seinem Entschluss zur Kostenübernahme [15] der operativen Behandlung des Lipödems vom Sommer 2017 entschieden, dass vor einer endgültigen Entscheidung zur Bewertung der operativen Behandlung des Lipödems eine Multicenterstudie durchgeführt werden solle. Entscheidendes Kriterium für den Erfolg der Methode soll dabei der Einfluss auf den Schmerz sein. Die Richtlinie zur Durchführung der Erprobungsstudie [16] wurde dann durch den G-BA im Januar 2018 beschlossen. Eine Bestätigung dieses Entschlusses erfolgte dann im April 2018 durch das Bundesgesundheitsministerium [16]. In diesem Entschluss wird das Krankheitsbild orientierend dargelegt,
- Tab. 1 Diagnostische Kriterien, modifiziert in Anlehnung an die S1-Leitlinie der AWMF [3].

Beginn mit der Pubertät oder Schwangerschaft oder Menopause

familiäre Häufung

die Veränderungen des Lipödems treten immer symmetrisch auf

disproportionale Fettgewebsvermehrung (Extremitäten > Stamm)

Kragenbildung im Bereich der Gelenkregionen

Schwere- und Schwellgefühl der betroffenen Extremitäten

Schmerzhaftigkeit bei Palpation oder spontan - im Tagesverlauf zunehmend

Neigung zu Blutergussbildungen

Hände und Füße beschwerdefrei, Stemmer-Zeichen negativ

einschließlich der seit 2017 vorliegenden ICD-Codierung durch die Ziffern E88.20 bis 22. Endpunkt der durchzuführenden Untersuchung soll dabei das Symptom „Schmerz“ sein.

\section{Zitat aus der Erprobungsstudie des G-BA}

Das Ziel der Erprobungsstudie besteht darin, die möglichen Vorund Nachteile der Liposuktion gegenüber der alleinigen KPE des Lipödems mit hinreichender Genauigkeit zeigen zu können. Ziel der Behandlung des Lipödems wiederum ist die Reduktion der Beeinträchtigung durch die Symptome. Analog zu den in den bisherigen Studien berichteten wesentlichen Symptomen des Lipödems wurde Schmerz als primärer Endpunkt ausgewählt [16].

Ziel der vorliegenden Untersuchung war es, nicht nur den Schmerz nochmals genauer zu charakterisieren, sondern zusätzlich auch noch die durch die Symptomatik hervorgerufene Beeinträchtigung im Alltagsleben bis hin zur Beeinträchtigung der Arbeitsfähigkeit zu erforschen.

\section{Methodik/Patienten}

Im Zeitraum von Januar 2017 bis Juli 2017 wurde allen Patientinnen, die sich zur Diagnostik bzgl. des Vorliegens eines Lipödems vorstellten, der Schmerzfragebogen (DSF) [17] in der langen Version der Deutschen Schmerzgesellschaft e. V. vorgelegt. Zu den Einzelheiten des Schmerzfragebogens wird auf die Veröffentlichung der Deutschen Schmerzgesellschaft e. V. im Internet unter https://www. dgss.org/schmerzfragebogen/ verwiesen ( $\bullet$ Abb. 1). Sämtliche Untersuchungen wurden ausschließlich durch den Autor und den Koautor persönlich vorgenommen. In der Auswertung wurden jedoch nur die Fragebögen berücksichtigt, bei denen bei den Patientinnen auch ein Lipödem diagnostiziert wurde. Unberücksichtigt blieb, ob die Patientinnen schon eine konservative Behandlung erfahren hatten oder die Absicht hatten, sich einer operativen Therapie zu unterziehen. In unserer Klinik erfolgt sowohl eine konservative als auch operative Behandlung. Bei der Auswertung wurde nicht berücksichtigt, welche Folgetherapie durchgeführt wurde. Die Auswertung erfolgte mittels der Software Akkaya Consulting. Insgesamt konnten 640 Fragebögen berücksichtigt werden. In den berücksichtigten 


\section{Deutscher Schmerz-Fragebogen}
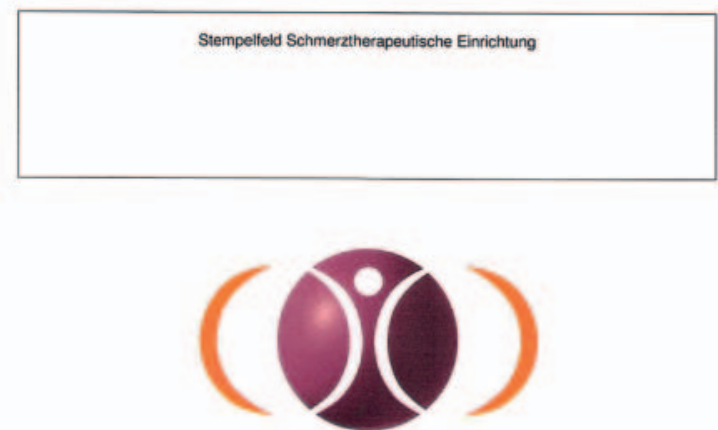

\section{Deutsche Schmerzgesellschaft e.V.} Sektion der International Association for the Study of Pain (IASP)

Copyright: www.dgss.org

Version 2015.2

Juni 2015
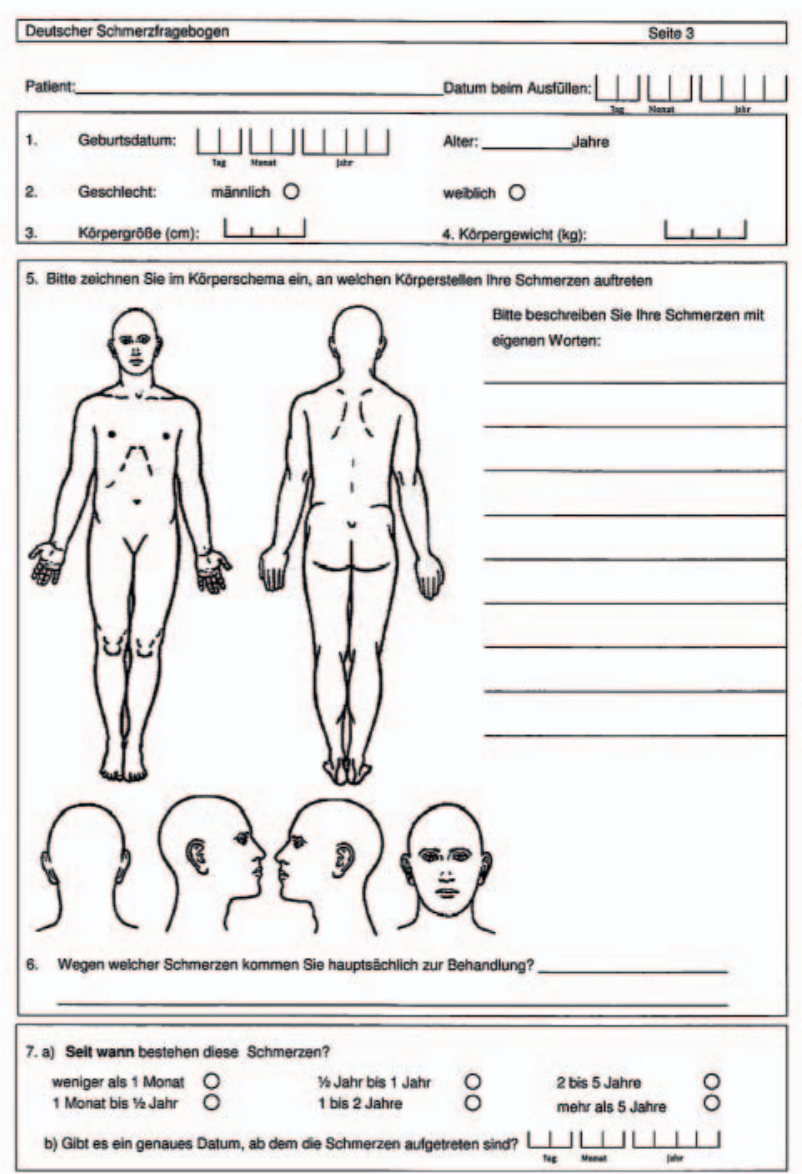

- Abb. 1 Deckblatt Fragebogen, exemplarisch Seite 3 von 14.

Fragebögen wurden jedoch nicht alle Fragen durch alle Patientinnen beantwortet, sodass bei der Auswertung (s. Abbildungen) zum Teil unterschiedliche Ergebniszahlen zu finden sind.

\section{Ergebnisse}

Mehr als die Hälfte der befragten Patientinnen waren im Alter zwischen 30 und 49 Jahren, die jüngste Patientin, die sich vorstellte, war 15 Jahre alt, die älteste Patientin hatte das 80. Lebensjahr vollendet ( $>$ Abb. 2 ).

Das beim Lipödem immer wieder aufgeführte Übergewicht fand sich bei etwas über 50 \% der befragten Patientinnen, die übrigen Patientinnen - und hierbei von großer Bedeutung - waren völlig normalgewichtig oder als leicht adipös (BMI <30) zu bezeichnen ( $\triangleright$ Abb. 3). Über 20 \% der Patientinnen hatten einen BMI, der völlig im Normbereich lag. Die durch das Lipödem bei der Untersuchung durch die Autoren ausgelöste Schmerzsymptomatik zeigte sich dabei gewichtsunabhängig.

Bei nahezu 50 \% der befragten Patientinnen liegt die Schmerzsymptomatik schon mehr als 5 Jahre vor. Die Schmerzhaftigkeit unterliegt dabei einem zirkadianen Rhythmus mit einem Maximum der Symptomatik im Laufe des Abends ( $\mathbf{A} \mathbf{b b} \mathbf{4} \mathbf{4})$.

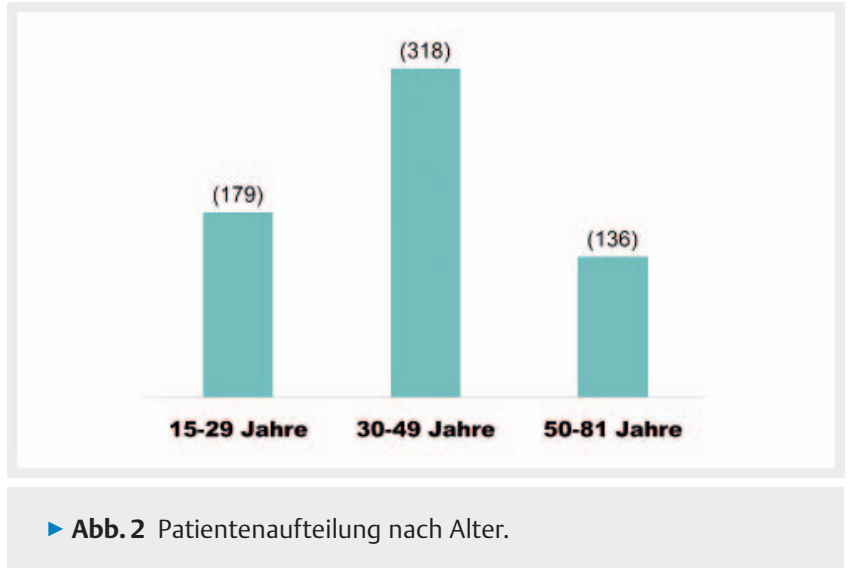

Der verwendete Fragebogen ermöglichte eine Vielzahl von Charakterisierungen des Symptoms „Schmerz“. Im Folgenden findet sich ein Übersichtsschaubild der gefundenen Beschreibungen $(\triangleright$ Abb. 5).

Die verschiedenen Schmerzbeschreibungen konnten von „genau zutreffend“ bis „nicht zutreffend“ angekreuzt werden. Weit über 50 \% der Patientinnen gaben dabei den Schmerz als drückend an, am zweithäufigsten wurde der Schmerz als ziehend 


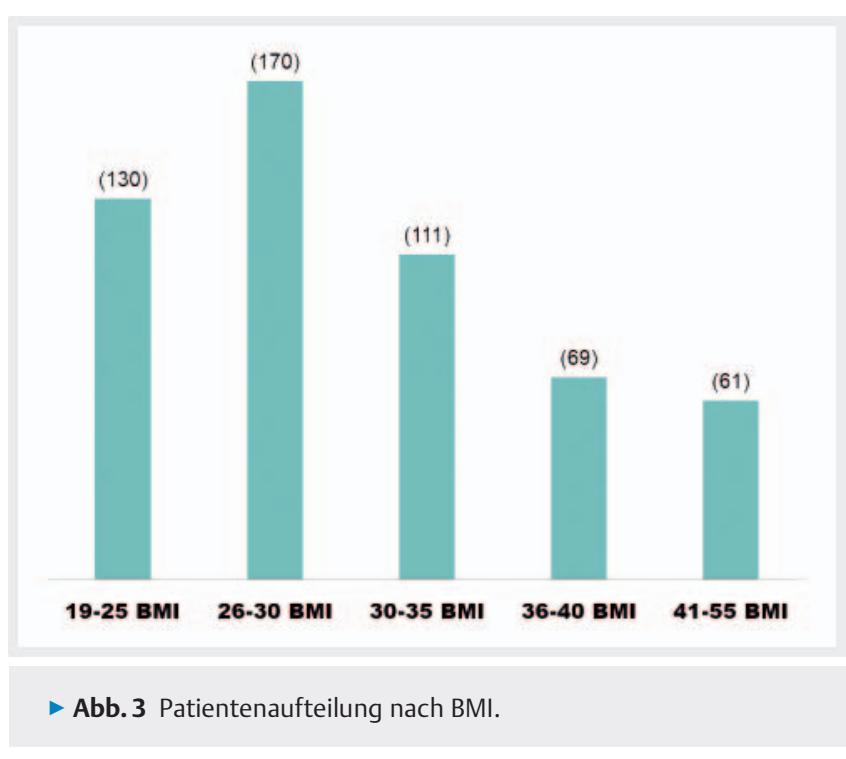

beschrieben. Im Gegensatz hierzu wurde von der Mehrheit der Patientinnen angegeben, dass die Schmerzsymptomatik keinesfalls klopfend, wie z. B. bei einer Entzündungsreaktion, empfunden wurde. Die Einzelheiten sind in den beiden folgenden Schaubildern grafisch wiedergegeben. ( $>$ Abb. 6, 7)

Ca. $90 \%$ der Patientinnen gaben tägliche Schmerzen an. Etwa $50 \%$ der Patientinnen gaben dabei eine mittlere Intensität der Schmerzsensation zwischen Stufe 2 und Stufe 6 auf der Intensitätsskala von 0-10 an. Ein Großteil der Patientinnen war dabei für Verrichtungen des Alltags beeinträchtigt. Die Beeinträchtigung lag überwiegend im Rang zwischen 2 und 8 ( $>$ Abb. 8).

Eine ähnliche Verteilung und Beeinträchtigung fand sich auch für die Freizeitaktivitäten. Diese Feststellung darf keinesfalls zu dem Schluss führen, dass $21 \%$ der Patientinnen ( $\boldsymbol{\text { Abb. }}$ 8) keine Schmerzen angaben, sondern nur, dass sie sich nicht durch die Schmerzsymptomatik beeinträchtigt fühlten. Die konservative Behandlung mittels KPE kann ja zumindest zu einer Schmerzreduktion [18] führen, was sich auch mit eigenen Beobachtungen deckt.

In einer kleinen Minderheit (ca. $3 \%$ ) ergab sich sogar eine vollständige Beeinträchtigung der Arbeitsfähigkeit, die in eine Arbeitsunfähigkeit mündete.

Die Schmerzsymptomatik führte dazu, dass über $80 \%$ der Patientinnen angaben, in unregelmäßigen Abständen Schmerzmedikamente einzunehmen. In erster Linie wurden dann jedoch nichtsteroidale Antirheumatika eingenommen, an erster Stelle wurde dabei Ibuprofen in der Dosierung von $600 \mathrm{mg}$ genannt. Regelmäßige Medikamenteneinnahmen stellten die Ausnahme dar, zumal diese Medikamente nicht zu einer dauerhaften Schmerzreduktion führten.

Trotz der Beeinträchtigungen werteten über $50 \%$ der befragten Patientinnen ihren Allgemeinzustand zumindest als gut, sehr gut oder sogar ausgezeichnet.

\section{Diskussion}

Der verwendete Fragebogen der DGFS sollte eine Nachvollziehbarkeit der Untersuchungsergebnisse oder weiterführender

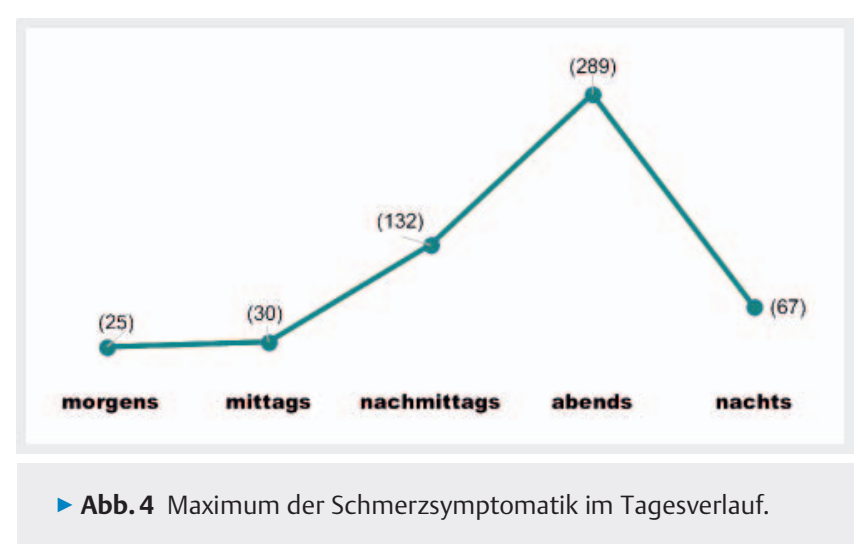

Untersuchungen ermöglichen. Es zeigte sich aber bei der Auswertung, dass eine ganze Reihe von Fragen bei Lipödem-Patientinnen nur bedingt Anwendung finden konnten. Dies führte auch dazu, dass einige Patientinnen die Fragebögen nicht vollständig ausfüllten. Einige Fragestellungen, wie z. B. die Korrelation der Stadien des Lipödems zu den aufgeführten Veränderungen der Lebensqualität, ließen sich nicht durch die verwendete Auswertungssoftware beantworten. Bei weiteren Untersuchungen ist es nach Meinung der Autoren daher sinnvoll, einen modifizierten, auf das Lipödem besser zutreffenden Fragebogen zu entwickeln.

Es ist keinesfalls so, dass Patientinnen mit einem höheren BMI im Rahmen der Untersuchung eine ausgeprägtere Schmerzsymptomatik aufwiesen. Auch diese Differenzierung ließ der verwendete Fragebogen nicht zu.

Die Daten zeigen auf, dass die Diagnose Lipödem nicht zwingend Adipositas-assoziiert ist, auch wenn die derzeitige Diskussion in diese Richtung zielt. Laut einer Untersuchung der Deutschen Gesellschaft für Ernährung sind am Ende ihres Berufslebens 74,2\% der Männer übergewichtig, bei Frauen im gleichen Alter sind es 56,3 \% [19]. Die Bewertung des BMI ist bei Patientinnen mit Lipödem aber nur bedingt verwertbar, da Patientinnen bei diesem Krankheitsbild sehr wohl ihre Adipositas, sofern sie denn vorliegt, reduzieren können; einen wesentlichen Einfluss auf die durch das Lipödem veränderten Extremitäten und die Schmerzsymptomatik hat die Gewichtsreduktion jedoch nicht. Im Rahmen der LipödemSprechstunde in unserer Klinik werden durch uns immer wieder Patientinnen gesehen, die bei einer morbiden Adipositas einer bariatrischen Operation zugeführt wurden und dann 50-60 kg abnahmen.Die massive Fettverteilungsstörung der Extremitäten verblieb jedoch bei Patientinnen mit gleichzeitig bestehendem Lipödem. Die Aussage von Patientinnen „Ich kann nicht abnehmen.“ müsste also korrekt lauten „Ich kann mein Lipödemfett nicht abnehmen." Dies steht auch im Einklang mit der in der AWMF-Leitlinie getroffenen Schätzung, dass nur etwa 6-8\% der weiblichen Bevölkerung in Deutschland von diesem Krankheitsbild betroffen sind [20].

Die Daten zeigen, dass das Symptom „Schmerz“ extrem facettenreich ist. Unter diesem Aspekt muss die durch den G-BA initiierte Studie zum Lipödem kritisch gesehen werden, in der als Endpunkt Schmerz als Hauptkriterium für den Erfolg der konservativen oder operativen Behandlung vorgesehen ist. Ziel aller Behandlungsmaßnahmen muss die Schmerzfreiheit oder zumin- 


\section{Mit der folgenden Liste von Eigenschaftsworten können Sie genauer beschreiben, wie Sie lhre Schmerzen empfinden. Denken Sie bei der Beantwortung an Ihre typischen Schmerzen in der letzten Zeit.}

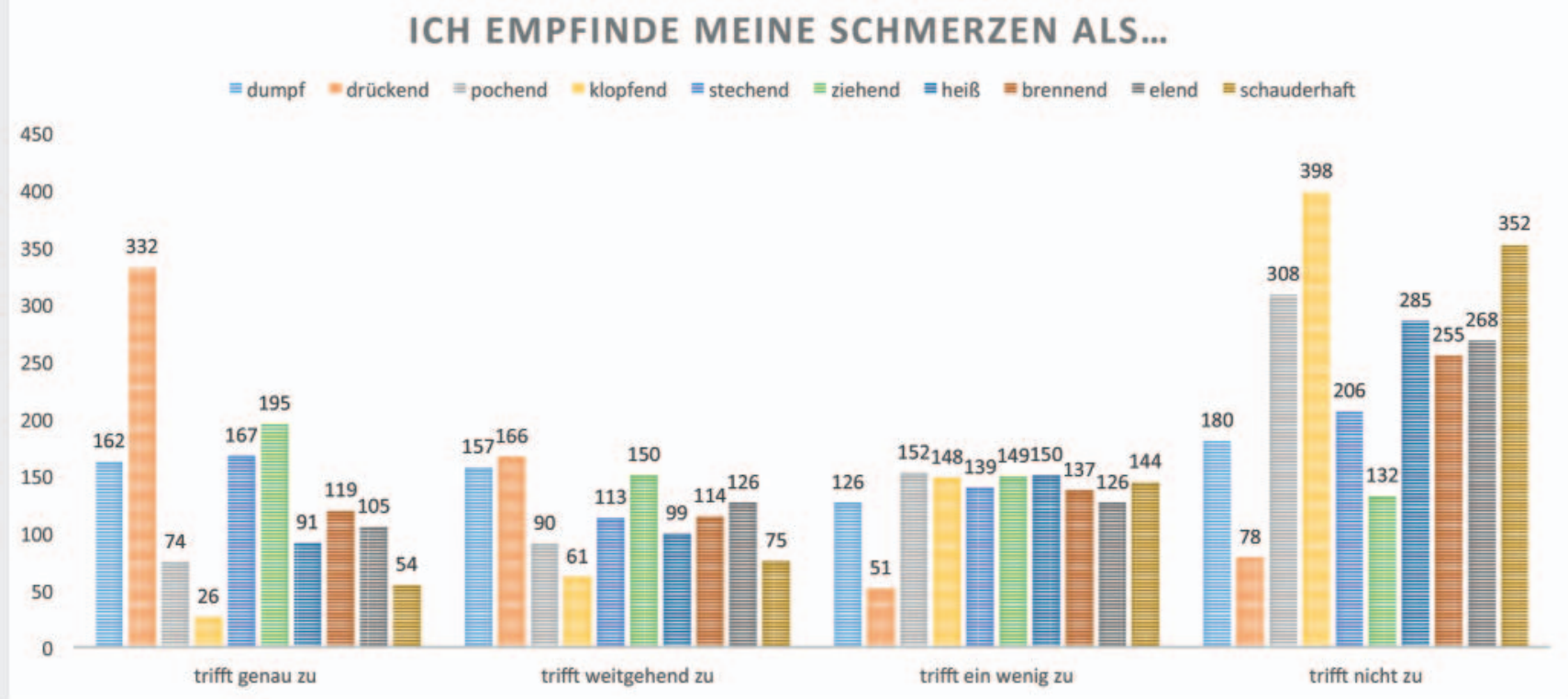

- Abb. 5 Übersicht: Subjektive Charakterisierung des Lipödemschmerzes.

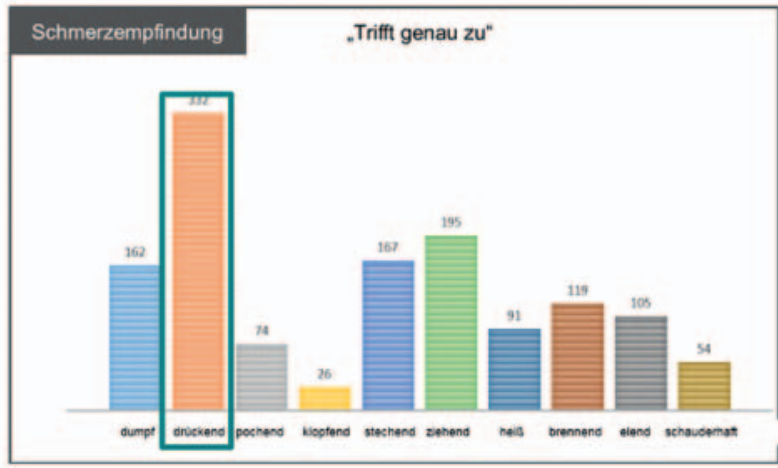

Abb. 6 Detailansicht: Subjektive Charakterisierung des Lipödemschmerzes.

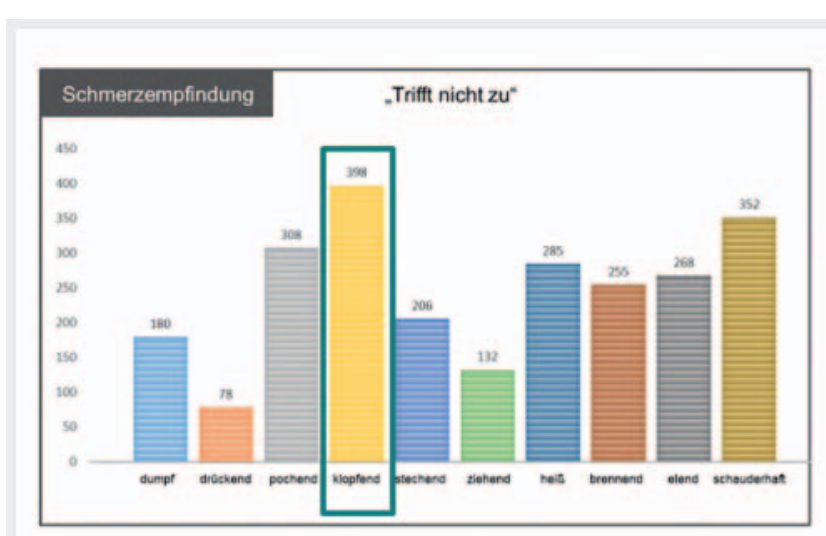

- Abb. 7 Detailansicht: Subjektive Charakterisierung des Lipödemschmerzes. dest weitgehende Schmerzfreiheit sein. Weiterhin sollte auch keine Therapiebedürftigkeit, weder Lymphdrainage noch Kompressionsbehandlung, in der Folgezeit erforderlich sein. Nach Möglichkeit muss das Ziel eine Heilung und damit eine Rezidiv-Freiheit bei den Behandlungen sein. Der vorgesehene Zeitraum der Studie des G-BA ist kaum dazu angetan, diese Kriterien beurteilen zu können. Die meisten Patientinnen gaben an, dass der Schmerz dumpf, drückend, ziehend und stechend sei. Dennoch waren auch noch weitere Attribute für die Schmerzempfindung gegeben. Die Attribute klopfend und pochend, Attribute, die typischerweise bei Entzündungsreaktionen gefunden werden können, sind jedoch deut- lich geringer angegeben worden, dafür in der Rubrik „nicht zutreffend“ besonders häufig genannt worden. Dies lässt den Schluss zu, dass es sich bei der Schmerzsymptomatik des Lipödems nicht um einen Entzündungsprozess handelt. Auch wenn im Rahmen der Untersuchung keine Laboruntersuchungen stattfanden, steht dies im Einklang mit den Labordaten wie BSG, Leukozytenzahl und CRP, die regelmäßig im Rahmen der präoperativen Blutuntersuchungen der in unserer Klinik operierten Patientinnen erhoben werden. Die Entzündungsparameter sind in aller Regel unauffällig. Auffällig ist jedoch die sehr unterschiedliche Intensität des Schmerzes. Der Schmerz kann dabei spontan 


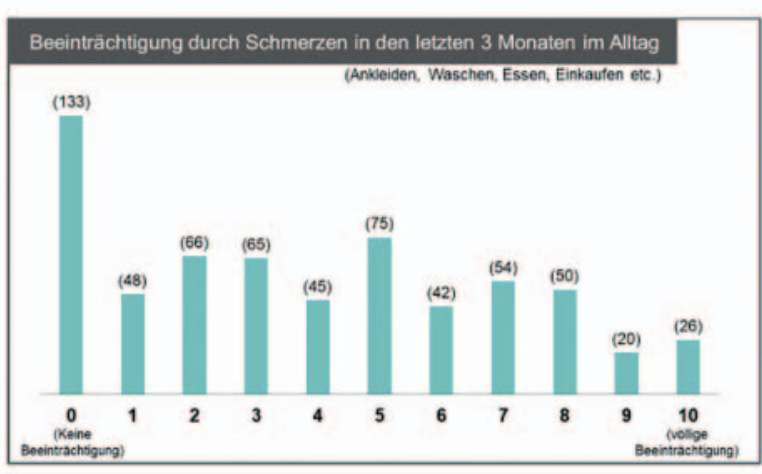

- Abb. 8 Detailansicht: Subjektive Beeinträchtigung durch Lipödemschmerz.

auftreten, aber auch erst auf äußere Reize ausgelöst werden. Im Rahmen der Diagnostik bedarf es also einer sehr subtilen Befragung der Patientinnen, damit das Leitsymptom des Lipohyperplasia dolorosa - der Schmerz - ausreichend und korrekt gewürdigt wird. Die sorgfältige gezielte Anamnese ist somit eine wesentliche Voraussetzung für eine korrekte Diagnosestellung. Suggestivfragen sollten jedoch vermieden werden. Die demografischen Daten, die im Rahmen der Befragung erhoben wurden, zeigen deutlich, dass die immer wieder erwähnte Adipositas nur als Komorbidität bei $50 \%$ der befragten Patientinnen nachgewiesen wurde [21]. Insofern ist es mehr als fragwürdig, dass die z. Zt. im Rahmen von Kongressen geführte Diskussion beim Lipödem fast ausschließlich von einem Adipositas-assoziiertem Krankheitsbild spricht. Auch wenn die Anzahl der adipösen Patientinnen annähernd 50 \% beträgt, müssen Lipödem und Adipositas als 2 getrennte Krankheitsbilder betrachtet werden. Die immer wieder geäußerte Behauptung, Lipödem-Patientinnen könnten nicht abnehmen, ist so nicht korrekt. Lipödem-Patientinnen können abnehmen, aber eben nicht das an den Extremitäten ausgeprägte, nicht erworbene, sondern angeborene genetisch bedingte druckschmerzhafte Lipödemfett. Begleitend führt dies zu deutlich nachweisbaren Beeinträchtigungen im täglichen Leben, sowohl was die allgemeine Beweglichkeit anbelangt als auch das, was als Lebensqualität angesehen wird. Die Daten zeigen, dass von geringfügigen Beeinträchtigungen im täglichen Leben sowohl was den Arbeitsprozess - auch die Hausarbeit - betrifft als auch die Möglichkeiten der Freizeitgestaltung über 85 \% der LipödemPatientinnen betroffen sind. Aufgrund dieser doch erheblichen umfassenden Beeinträchtigung der Lipödem-Patientinnen sollte daher durch die möglichen Behandlungsoptionen, konservativ oder operativ, das vorrangige Ziel sein, das Lipödem in der Beschwerdesymptomatik zu reduzieren oder durch Operationen zu kurieren. Auch wenn von Patientenaussagen unabhängige objektivierbare Befunde z. Zt. nicht erhebbar sind, sollten Patientinnen mit ihrem Krankheitsbild endlich ernst genommen werden.
Interessenkonflikt

Die Autorinnen/Autoren geben an, dass kein Interessenkonflikt besteht.

Literatur

[1] S1-Leitlinie Lipödem, AWMF-Register-Nr. 037-012.

[2] Allen E, Hines EA. Lipedema of the legs: A syndrome characterized by fat legs and orthostatic edema. Proc Staff Mayo Clin 1940; 15: 184-187

[3] Cornely ME, Schnabel H, Moser S et al. Diagnostik und Differentialdiagnostik des Lipödems. 1998

[4] Cornely ME. Lipödem und Lymphödem. In: Plewig G, Prinz J, (Hrsg) Fortschritte der praktischen Dermatologie und Venerologie. Berlin, Heidelberg, New York, Tokyo: Springer; 2003: 255-263

[5] Wiedner M, Aghajanzadeh D, Richter D. Lipödem-Grundlagen und aktuelle Thesen zum Pathomechanismus. Handchir Mikrochir PlastChir 2018; 50: $380-385$

[6] Zetzmann K, Ludolph I, Horch R et al. Bildgebende Diagnostik zur Therapieplanung beim Lip- und Lymphödem. Handchir Mikrochir PlastChir 2018; 50: 386-392

[7] Koban K, Titze V, Etzel L et al. Quantitative volumetrische Analyse der unteren Extremität: Validierung gegenüber etablierter Messbandmessung und Wasserverdrängung. Handchir Mikrochir PlastChir 2018; 50: 393-399

[8] Tiedjen KU, Heimann KD, Tiedjen-Kraft U. Indirect Xero-lymphography in lymphedema, lipedema and venous insufficiency. In: RaymondMarinbeau P, Prescott R, Zummo M, (Hrsg) Phlebologie. Paris: John Libbey Eurotext; 1992: 396-398

[9] Cornely ME. Zur Terminologie bei Lipödem. MÄC 4. 2004; 4

[10] Marsch WCH. Ist das Lipödem ein lymphologisches Krankheitsbild? J Lymphol 2001; 1: 22-24

[11] Marsch WCH. Lymphgefäßsystem und Haut. Orthologie, Klinik und Pathologie. Hautarzt 2005; 56: 277-295

[12] Cornely ME. Das Lipödem an Armen und Beinen Teil 1: Pathophysiologie Phlebologie. 2011

[13] Schmeller W, Meier-Vollrath I. Schmerzen beim Lipödem, Versuch einer Annäherung LymphForsch12 (1) 2008.

[14] Brenner E. Wie kommt der Schmerz ins Lipödem. LymphForsch 2017; 21 (1): 40-47

[15] Beschluss des Gemeinsamen Bundesausschusses über eine Änderung der Richtlinie Methoden Krankenhausbehandlung: Liposuktion bei Lipödem 20. Juli 2017, BAnz AT 17.10.2017 B3.

[16] Beschluss des Gemeinsamen Bundesausschusses über eine Richtlinie zur Erprobung der Liposuktion beim Lipödem, BAnz AT 09.04.2018 B1.

[17] Deutsche Schmerzgesellschaft e. V.: Deutscher Schmerzfragebogen. Stand: Juni 2015. Im Internet (Stand: 19.06.2018): https://www.dgss. org/schmerzfragebogen/

[18] Brenke R et al. Klinische Erfolge der komplexen Entstauungstherapie beim Lipödem, ViavitalKöln 2001. 215-217

[19] Presseinformation: Presse, DGE aktuell, 2017 03/2017 vom 01.02.2017.

[20] AWMF-Register-Nr.: 037-012/Stand: 31.10.2015/gültig bis: 30.06 .2020

[21] Cornely ME. Dicker durch Fett oder Wasser. Hautarzt 2010; 61: 873-879 